\title{
Virgilio e la strumentalizzazione della ragione nell'Inferno di Peter Weiss
}

\author{
Carlota Cattermole Ordóńez \\ Universidad Complutense de Madrid \\ carlota.cattermole@gmail.com
}

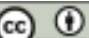

\begin{abstract}
L'articolo propone un'approssimazione all'Inferno (2003) di Peter Weiss, una riscrittura drammatica in trentatré canti della prima cantica della Commedia, che è rimasta inedita dall'anno della sua redazione (1964) come conseguenza dell'abbandono - temporaneo - del cosiddetto DC-Projekt, di cui faceva parte. Il mio contributo si centra nell'analisi del personaggio di Virgilio con l'obiettivo di offrire una lettura capace di rendere conto dei processi di attualizzazione che subiscono i materiali originali per essere reintrodotti, con significati rinnovati, nel testo derivativo. Si dimostra come, nonostante l'apparente lontananza dall'ipotesto, l'Inferno di Peter Weiss rinvii in maniera costante alla Commedia e come il riconoscimento dei nascosti riferimenti al modello scopra delle dimensioni del testo inavvertite. In concreto, l'articolo suggerisce, dopo una necessaria presentazione e contestualizzazione dell'opera, una nuova interpretazione di Virgilio, personaggio che metto in relazione con il concetto di ragione strumentale elaborato da Adorno e Horkheimer nella loro Dialektik der Aufklärung (1947).
\end{abstract}

Parole chiave: Peter Weiss, DC-Projekt, trilogia drammatica, Virgilio, intertestualità, Auschwitz, ragione strumentale.

\begin{abstract}
The article proposes an approach to Inferno (2003) by Peter Weiss, an atypical dramatic rewriting of thirty three cantos of the first Canto of the Commedia that has remained untouched since the year of its composition (1964) as a result of the contemporary neglect of the so-called DC-Projekt of which it was a part. My contribution focuses on the analysis of the character of Virgil with the aim to offer a reading capable of understanding the processes of transformation of the original materials in order to be presented again with a renewed meaning of the text from which it derives. Despite the apparent distance of the hypotext, l'Inferno of Peter Weiss makes constant references to the Commedia and acknowledges hidden references to the model by revealing dimensions that have gone unnoticed. After a necessary presentation and contextualization of the play, what the article concretely suggests, is a new interpretation of Virgilio through its relation to the concept of instrumental rationality made by Adorno and Horkheimer at their Dialektik der Aufklärung (1947).
\end{abstract}

Key words: Peter Weiss, DC-Projekt, Dramatic Trilogy, Virgil, Intertextuality, Auschwitz, instrumental reason. 
66 D robabilmente nessun'altra opera all'interno della letteratura tedesca 1 permette di osservare in presa diretta il tentativo e l'impegno di un autore di fare propria un'altra opera e amalgamarla alla propria come nel caso del 'Progetto-Dante' (Dante-Projekt) di Peter Weiss" (Dallapiazza 20I0: 135). Solo Pasolini ${ }^{1}$ raggiunge, infatti, la "obsessive Fixierung" (Kuon 1997: 42) con cui lo scrittore tedesco (1916-1982) cerca di assimilare e attualizzare i contenuti danteschi in ognuno dei momenti che attraversano il suo ambizioso progetto, dalla prima fase concezionale del 1964 al suo definitivo abbandono nel 1969, dopo un ultimo tentativo, anch'esso fallito, di rifacimento in prosa del modello dantesco. L'eccezionalità del progetto weissiano di riscrittura della Divina Commedia è stata ormai sottolineata in non poche occasioni negli studi che si occupano del "dantismo" dello scrittore, ambito di ricerca che ha ricevuto nuovi impulsi dalla postuma pubblicazione di Inferno (2003) ${ }^{2}$. La scoperta del dramma, ritrovato da Christoph Weiß tra i materiali che si conservano nell'archivio di letteratura dell'Accademia delle Arti di Berlino (da ora in poi $A d K)^{3}$ durante la sua ricerca sulla genesi e ricezione dell'Istruttoria ${ }^{4}$, segna infatti uno spartiacque nell'ambito della filologia e critica weissiana, non solo perché esige il ripensamento degli studi realizzati fino ad allora ma anche - e soprattutto - perché "l'attenta analisi del lavoro su Dante, di cui Inferno è una tappa fondamentale, apre a una riconsiderazione dell'ormai obsoleto paradigma critico che suddivideva la scrittura weissiana in una prima fase 'soggettiva' e una seconda fase 'politica"' (Castellari 2008: 252).

Ricollegabile alla prima fase del DC-Projekt $t^{5}$, che prevedeva il rifacimento drammatico delle tre cantiche della Divina Commedia, l'Inferno è un chiaro

I. Per una comparazione dei progetti di Pasolini e Peter Weiss si veda Kuon (I993: 302-37I), Kuon (1997) e Wöhl (1997).

2. La pubblicazione dell' Inferno ha avuto però scarsa ripercussione sia tra i lettori che nel contesto teatrale. La première dell'Inferno si svolge nella versione operistica di Johannes Kalitzke l'II giugno del 2005 a Brema (si veda Heinrich 2006). Sotto la direzione di Thomas Krupa si realizza poi la prima rappresentazione teatrale del dramma, il 26 gennaio del 2008 a Karlsruhe (Si veda Weymann 2008; Kretschmann 20I2).

3. Per citare le cartelle che si trovano nell'archivio di letteratura si rispetta il metodo per cui dopo la sigla PWA (Peter Weiss Archiv) si fornisce il numero della cartella e la pagina.

4. Christoph Weiß dedica proprio all'Inferno un intero capitolo del suo studio sulla genesi dell'Istruttoria e sulla sua polemica ricezione nella Germania della Guerra Fredda. Nel tentativo di contestualizzare il dramma dentro del cosiddetto DC-Projekt e di scoprire quale legame esista tra Istruttoria e Inferno, egli fornisce il primo riferimento all'opera recentemente scoperta (Weiß 2000). Una versione ridotta dell'articolo, nella traduzione italiana di Marco Castellari, si trova nell'edizione italiana dell'opera (Weiss 2008).

5. La prima fase del progetto si svolge tra febbraio del 1964 e marzo del 1965 . È stato Yannick Müllender a ricostruire i diversi momenti del DC-Projekt nella sua tesi di dottorato, pubblicata nel 2007. Risultato di un titanico lavoro filologico, il volume ricostruisce le diverse fasi del tortuoso viaggio weissiano attraverso la Commedia e include sia un dettagliato stemma codicum in cui si spiegano i rapporti genetici che legano i manoscritti sia 
esempio di come gran parte dei fenomeni di ricezione produttiva dell'opera di Dante nel ventesimo secolo si articolano attorno alla tensione che si stabilisce tra i due poli dell'inattualità della Commedia - sia essa avvertita come distanza storica, ideologica, linguistica o formale - e dell'attualità delle circostanze biografiche ed esistenziali del suo autore (Kuon 1997: 47), che diventa modello dello scrittore impegnato, con cui molti intellettuali condividono la condizione di exul immeritus. L'intenso dialogo che Weiss stabilisce con Dante si nutre in maniera particolarmente evidente di questa contraddizione: da una parte il protagonista del dramma Inferno è un personaggio di nome Dante che, in quanto alter ego dell'autore, ritorna in patria dopo la traumatica esperienza dell'esilio $^{6}$, dall'altra, la percezione dell'estraneità del modello esige il ribaltamento della sua struttura originale. La negazione dei contenuti danteschi si configura infatti più come una necessità che come una scelta liberamente adottata dallo scrittore tedesco, cosciente del fatto che l'unica possibilità di superare la distanza ideologica che lo separa dalla Commedia risieda nella radicale trasformazione dei suoi significati originali7.

Questa distanza diventa specialmente evidente dopo la frattura di Auschwitz: l'insensatezza della morte, la distruzione dell'uomo come soggetto morale e giuridico e l'esistenza stessa di spazi di indeterminazione come quello descritto da Primo Levi con il concetto di 'zona grigia' mettono in evidenza come il lager - dove "liegen doch die Unschuldigen" (Weiss 1968: 146) [si trovano però gli innocenti] - rappresenti l'antitesi stessa dell'Inferno dantesco, una costruzione razionale, giusta e strutturata in termini etici che derivano direttamente dall'intervento divino - "Giustizia mosse il mio alto fattore" (If. III 4). La legge del contrappasso, che nella Commedia - non a caso definita da Weiss "Weltgericht" (Weiss 1982: I2) - regola la corrispondenza tra colpa e castigo, non ha più validità nella società del dopo Auschwitz, dove i colpevoli restano "impuniti" e le vittime inascoltate. Si tratta di un'incompatibilità che lo scrittore traduce invertendo la struttura del poema e secolarizzando i tre regni ultramondani, come spiega nel Vorübung zum dreiteiligen Drama

un capitolo dove si possono leggere i testi editati - schemi, frammenti, bozze, e versioni alternative - che si conservano nell'archivio dell'AdK di Berlino.

6. L'affinità biografica con Dante è stata esplicitamente notata da Weiss nei suoi metatesti, in particolare nel Gespräch über Dante (1965) [Dialogo su Dante]. Conviene ricordare che nell'anno 1939 comincia l'esilio della famiglia di radici ebraiche Weiss che si rivelarà definitivo per l'autore che si stabilirà definitivamente in Svezia. Ciononostante il progetto di riscrittura della Divina Commedia è indissolubilmente legato alla realtà tedesca del dopoguerra ed è precisamente durante un lungo soggiorno a Berlino occidentale, nella primavera del 1964, che il progetto comincia a prendere forma.

7. "Was konnten mir Dantes Begriffe geben, diese Einteilungen in Aufenthaltsorte für Büßende, Erlöste und selig Belohnte? Dies alles war meiner eigenen Welt entgegengesetzt" (Weiss 1968: 132) [Che potevano darmi i concetti di Dante, quella suddivisione in luoghi dove dimoravano i penitenti, i redenti e i beati? Tutto ciò era all'opposto del mio mondo] (Weiss 2007: 34). 
divina commedia (1965) [Esercizio preliminare per il dramma in tre parti divina commedia]:

Dante, sollte er seine Wanderung noch einmal antreten, müßte nach anderen Mitteln suchen, seine Zeit zu vergegenwärtigen, grundlegend müßte er den Sinn revidieren, den er den Ortschaften Inferno, Purgatorio und Paradiso beigemessen hatte [...] Das Grundmuster zeigte sich wie folgt: Inferno beherbergt alle die, die nach des früheren Dante Ansicht zur unendlichen Strafe verurteilt wurden, die heute aber hier weilen, zwischen uns, den Lebendigen, und unbestraft ihre Taten weiterführen, und zufrieden leben mit ihren Taten, unbescholten, von vielen bewundert. [...] Purgatorio dann ist di Gegend des Zweifelns, des Irrens, der mißglückten Bemühungen, die Gegend des Wankelmuts und des ewigen Zwiespalts [...]. Deutlich sah ich des Paradiso, wo jene zuhause sind, denen Dante einmal Glückseligkeit zusprach $^{8}$ (Weiss I968: I36-I38).

In questo modo, Peter Weiss rinnova e capovolge una delle tendenze che in maniera più evidente hanno definito la ricezione della Commedia nel XX secolo: l'appropriazione dell'immaginario dantesco come strumento di comparazione per descrivere l'indicibile dei campi di concentramento e sterminio, "una realtà che ha superato gli orrori tradizionali dell'Inferno" (Casadei 20I3: I5I), è abituale sia nella cosiddetta letteratura concentrazionaria, come ha dimostrato T. Taterka (I999) ${ }^{9}$, che nelle approssimazioni filosofiche, sociologiche e storiche al fenomeno.

Peter Weiss nega invece la validità di questo fecondo uso del testo dantesco e preferisce identificare l'Inferno con la Germania federale del miracolo economico (Wirtschaftswunder) dove il clima di euforia collettiva impedisce la rielaborazione critica della storia recente e dove sono ancora riconoscibili tracce nazionalsocialiste, sia a livello istituzionale sia ideologico. Nel dramma Inferno, unico testo che si conclude rispettando il progetto originale, Dante, che ritorna in patria dopo l'esilio incontra i persecutori di una volta, tutti personaggi provenienti dall'Inferno dantesco che conservano tratti della sua caratterizzazione originale e contemporaneamente si identificano, in maniera velata e mai definitiva, con referenti extra-testuali concreti, personalità del contesto politico e biografico dell'autore. I funzionari infernali, tali come Minosse, Gerione o il Minotauro, diventano rappresentanti del potere poli-

8. "Se intraprendesse il viaggio un'altra volta, Dante dovrebbe cercare altri mezzi per rievocare il proprio tempo, rivedendo radicalmente il senso che aveva attribuito alle località Inferno, Purgatorio e Paradiso. Lo schema di fondo si presentava così: L'Inferno ospita tutti quelli che secondo il Dante di una volta erano condannati a una pena infinita, e oggi, però, dimorano qui tra noi, i vivi, portando avanti impuniti i loro misfatti, incensurati, ammirati da molti. [...] Il Purgatorio, poi, è la terra del dubbio, dell'errore, dei tentativi falliti, la terra dell'irresolutezza e dell'eterno conflitto. [...]. Vedevo chiaramente il paesaggio del Paradiso, dove hanno casa quelli cui Dante un tempo assegnò la beatitudine" (Weiss 2007: 38).

9. Per approfondire tale argomento si veda anche Arqués 2009. 
tico ed economico nel dramma weissiano e, dopo il tentativo di integrazione del protagonista nella città di Dite, decretano violentemente il suo sterminio.

L'opera, come ha sottolineato per primo Brunner (2002), descrive inoltre l'evoluzione interiore del personaggio Dante: egli riesce a superare lo stato di iniziale paralisi di fronte alle accuse dei funzionari infernali, che strumentalizzano il suo sentimento di colpa e gli negano la condizione di vittima, e a sviluppare un atteggiamento critico nei confronti di una realtà infernale che si finge paradisiaca ${ }^{\mathrm{IO}}$. Nel mondo contemporaneo, impegnato a negare i crimini del passato, l'unico paradiso può essere però il luogo dove abitano i 'sommersi' di Auschwitz, scomparsi senza colpa. In nome loro testimoniano precisamente i superstiti del campo che intervengono appunto come personaggi nella polemica Die Ermittlung (1965) [Istruttoria in italiano] - originariamente $\mathrm{Pa}$ radiso -, opera con cui Peter Weiss aveva progettato di concludere la trilogia drammatica finalmente abbandonata. Si tratta del primo dei drammi documentali dello scrittore e nasce dallo studio e montaggio degli atti del Processo di Francoforte, alle cui sessioni aveva assistito lo stesso Weiss nell'aprile del 1964 ${ }^{\text {II }}$. L'istruttoria viene però svincolata definitivamente dal progetto di riscrittura della Divina Commedia, operazione che sancisce l'abbandono del piano originale e spiega quindi il carattere inedito dell'Inferno ${ }^{\mathrm{I} 2}$.

Il dramma Inferno risulta, come ha messo in luce Müllender (2007), da un tortuoso percorso redazionale di cui sono testimonianza le numerose bozze che si trovano nell'archivio, profondamente divergenti le une dalle altre non solo a livello concezionale e formale ma anche dal punto di vista del loro grado di emancipazione o di dipendenza dal modello. Elementi provenienti dalle versioni anteriori confluiscono in maniera apparentemente disordinata

IO. Cfr. Brunner 2002. È il ricordo di Beatrice, morta nel campo di sterminio, a tematizzare il complesso di colpa di Dante-Peter Weiss che non si sarebbe preoccupato di salvare l'amante di una volta e sarebbe invece fuggito in esilio.

II. Il ig ottobre del 1965 ha luogo la première dell'opera, che si svolge contemporaneamente in quattordici città tedesche, sia nella $\mathrm{Rft}$ che nella Rdt. L'intensa polemica suscitata dall'opera rappresenta una "Zäsur" per quanto riguarda il ripensamento del passato nazionalsocialista in Germania. Per la prima volta il dibattito si svolge parallelamente nelle due realtà tedesche della Guerra Fredda (Weiss 2000: 9).

I2. Subito dopo, Peter Weiss inaugura la seconda fase del DC-Projekt, a cui lavorerà tra i mesi di aprile del 1965 e febbrario del 1966 e da cui doveva risultare un Welttheater (teatro del mondo) non più centrato sulla storia della Germania ma atto a rispecchiare la complessità delle strutture di dominio e sfruttamento del mondo contemporaneo. L'universalizzazione delle riflessioni politiche è la caratteristica fondamentale di questa seconda fase del progetto, a cui si dedica Weiss precisamente nel periodo in cui il suo posizionamento pubblico in favore del socialismo sancisce la radicalizzazione della sua ideologia politica (sulle caratteristiche della seconda fase del DC-Projekt cfr. Müllender 2007: 205-244). 
nel testo definitivo, creando un dramma di enorme complessità strutturale e difficile classificazione che diventa specchio della sua stessa storia genetica.

Accanto a procedimenti di montaggio di questo tipo, l'introduzione di una cornice meta-teatrale di estetica grottesca e di effetti di straniamento di chiara eredità brechtiana - ottenuti attraverso l'impiego di maschere o l'intercambiabilità dei ruoli - rappresentano le novità formali più evidenti del testo definitivo. Ne emerge un dramma di enorme dinamicità che, anche a livello linguistico, è espressione di un universo schizofrenico e profondamente caotico ${ }^{13}$. Nella lettera con cui il lettore K. Braun, dell'editoriale Surkhamp, comunica a Weiss le impressioni ottenute dalla lettura del manoscritto dell'Inferno ${ }^{14}$, che lo scrittore gli aveva inviato con tale proposito nel mese di dicembre del 1964, l'opera viene duramente criticata precisamente per la sua incomprensibilità, riconducibile, a suo dire, soprattutto all'assenza di un intreccio riconoscibile e alla mescolanza intricata di piani temporali e spaziali. Braun espone inoltre il proprio disorientamento di fronte a un testo che già dal titolo stabilisce un legame stretto con la Divina Commedia, ma che contemporaneamente si allontana così radicalmente dal suo modello da provocare la confusione del lettore o dello spettatore.

Effettivamente, l'appropriazione weissiana dei materiali originali è sempre il risultato di un dialogo con il modello che si definisce a tutti i livelli in termini di contrapposizione e conflitto, per cui il testo è costruito in maniera che, contemporaneamente, cita e nega il proprio referente. Lo stesso Virgilio, uno dei personaggi che compaiono nel rifacimento weissiano, interviene nel canto IV del dramma per difendere la legittimità del capovolgimento dei significati originali ${ }^{15}$. Egli viene esortato da Caronte a spiegare a Dante personaggio quale sia il contenuto didattico implicito nel motto collocato sulla porta dell'Inferno, non a caso una delle testimonianze più radicali di tergiversazione, sia concettuale sia linguistica e metrica, del messaggio dantesco:

Auswechselbar verschiebbar je nach Wahl zu deuten / ist unser Werk / Wir greifen uns ein Ding heraus / stülpen es um und stelln es auf den Kopf / und schon ist es nicht wiederzuerkennen ${ }^{16}$ (Weiss 2008: 4I ${ }^{17}$

I3. Il testo è scritto infatti senza nessun tipo di punteggiatura e i versi non rispettano l'ordine abituale dei sintagmi della lingua tedesca.

14. Braun a Weiss I/I/1965 (PWA II3I).

15. "Per me si va nella città / che non è affatto dolente / per me si va dove tutto si raggiunge / Per me si va tra la gente che sempre / è vincente / Lasciate ogni dubbio voi ch'intrate" (Weiss 2008: 39).

I6. "Sostituibile differibile da interpretare a piacere / è la nostra opera / Scegliamo una cosa qualsiasi / la rivoltiamo e la capovolgiamo / e già non la si riconosce più".

17. Tutte le citazioni tratte dall'Inferno di Peter Weiss sono ricavate dall'edizione bilingue di Marco Castellari, di cui offriamo anche la traduzione italiana dei passaggi. 
Dall'intervento di Virgilio, che suggerisce al lettore una chiave interpretativa fondamentale, si evince infatti che la "tortura intertestuale" (Castellari 2008: 257) a cui è sottomesso il testo della Commedia contribuisce alla creazione di un dramma profondamente polisemico. La trasformazione dei contenuti e dei personaggi danteschi implica infatti la sopravvivenza più o meno esplicita di quei tratti dell'ipotesto che permettono di riconoscere la tergiversazione stessa. Dal mio punto di vista, è la memoria dell'originale a favorire la polisemia dell'opera, per cui l'ira di Flegiàs o la violenza del Minotauro, sono, per esempio, gli elementi che, rinviando contemporaneamente sia alla realtà del lager sia alla società tedesca del dopoguerra, suggeriscono l'identificazione dei due spazi. Una lettura di questo tipo, è consentita precisamente dall'assenza di referenti extra-testuali univoci e definitivi e dalla voluta indeterminazione dei riferimenti spaziali.

\section{III}

Il mio contributo propone un avvicinamento alla complessa caratterizzazione di Virgilio con l'obiettivo di dimostrare il protagonismo che acquista nel dramma la "questione intertestuale" (Castellari 2008: 257): una lettura attenta ai meccanismi con cui vengono attualizzati i contenuti originali, così come alle varianti presenti nelle diverse bozze, che sveli delle dimensioni del testo altrimenti impercettibili. In concreto, uno studio del genere permette di interpretare da un'ottica rinnovata l'atteggiamento ambiguo di Virgilio, conseguenza inevitabile della tensione che si stabilisce tra lo sforzo di attualizzare il ruolo che egli svolge nell'ipotesto e la consapevolezza delle difficoltà che questa operazione comporta. Dal mio punto di vista, il carattere contraddittorio del personaggio diventa espressione delle incertezze del suo autore, che progetta su di lui le proprie riflessioni intorno al ruolo della ragione nello scenario infernale del dopo Auschwitz.

A mio avviso, alcuni elementi della caratterizzazione di Virgilio rivelano infatti importanti coincidenze con il concetto di ragione strumentale sviluppato da Adorno e Horkheimer nella Dialektik der Aufklärung (1947), il che non significa che il personaggio si possa identificare in maniera a-problematica e univoca con esso. In effetti, la mia proposta di interpretazione non pretende né di esaurire la molteplicità di significazioni che il personaggio veicola né di dimostrare come Peter Weiss, attraverso una cosciente operazione di attualizzazione del significato allegorico del Virgilio dantesco, lo trasformi in rappresentazione della ragione strumentale. Intendo piuttosto mettere in luce una nuova dimensione del personaggio che non esclude necessariamente le altre ma che contribuisce invece a evidenziare la sua ricchezza significativa. 
Secondo me, ed è questo che mi propongo di mostrare nelle pagine seguenti, i contenuti della Dialettica dell'Illuminismo, o almeno il concetto di ragione che ne emerge, influiscono in modo più o meno cosciente sul processo di costruzione del Virgilio weissiano, determinando l'ambivalenza del suo comportamento nei confronti del protagonista del dramma. Ad appoggiare l'ipotesi che propongo, interviene, in primo luogo, una circostanza extratestuale che conferma come l'autore conoscesse il testo dei due filosofi: nei Notizbücher [Quaderni di appunti] originali compare infatti, nella pagina sessantotto del quaderno scritto tra il I5/12/1962 e il 20/04/1963, il titolo del volume (PWA 2082/68). Dal momento in cui nella Dialektik der Aufklärung si considera che sono la perversione e il fallimento del progetto dell'Illuminismo a provocare la decadenza della civiltà occidentale e i fenomeni di barbarie che ne derivano, è significativo, come giustificazione della nostra interpretazione, che Virgilio, in quanto rappresentante della ragione umana nell'ipotesto, sia vincolato da Weiss fin dalla fase concezionale del progetto alla possibilità di comprendere Auschwitz. Risulta chiarificatore ripercorrere le diverse bozze che precedono il dramma definitivo per capire in che termini si svolge la costruzione del personaggio e percepirne la mutevolezza.

Nella cartella 225I dell'archivio di letteratura (sezione Peter Weiss) dell'Accademia delle Arti di Berlino si conserva un manoscritto fondamentale per capire la genesi dell'Inferno. Si tratta di uno schema di lavoro che lo scrittore elabora in parallelo alla lettura e allo studio della Commedia, proponendo diverse modalità di attualizzazione dei contenuti originali e incorporando $\mathrm{i}$ versi che ritiene più significativi in forma di citazione.

Testimonianza privilegiata del processo creativo, lo schema evidenzia quali sono i punti che risultano più problematici per Peter Weiss. Espressioni come "noch nicht klar" (non ancora chiaro), "noch zu überlegen" (ancora da riflettere) o "stimmt das?" (è corretto?) sono, infatti, ricorrenti. È significativo, da questo punto di vista, che Virgilio sia l'unico personaggio dello schema di cui non si propongono uno o vari referenti extra-testuali. La sua caratterizzazione è senza termine di paragone quella più problematica per lo scrittore, incapace di definire il personaggio in modo univoco. Secondo le informazioni che possiamo dedurre dallo schema di lavoro, Peter Weiss progetta un dramma in cui Virgilio conserva il ruolo di guida che svolge nella Commedia. Prevede che sia proprio lui a convincere il poeta affinché continui il suo viaggio quando viene bloccato dalle tre fiere che gli rinfacciano il suo peccato di omissione. Virgilio mantiene la funzione esplicativa e protettiva dell'originale almeno fino al canto VII, momento in cui l'autore si mostra per la prima volta dubitativo circa la sua caratterizzazione. Interpreta come indicatore di un possibile tradimento nei confronti del pellegrino i versi (If. VIII, I09-II4) che manifestano 
lo sconforto con cui Dante vive l'abbandono di Virgilio, che si allontana per tentare di convincere i diavoli ad autorizzare la loro entrata nella città di Dite:

$\mathrm{Zu}$ Vergil: lass mich nicht allein ich fühle mich so hilflos 8/Ioo hier ist eine noch unklare Szene: Vergil tritt ein in DIS verhandelt dort, doch Dante erfährt nicht, was gesprochen wird. Vielleicht kann er auch an Vergil zweifeln verrät Vergil ihn am Ende an die Stadtbewohner. ${ }^{\text {?8 }}$ (Müllender 2007: 344)

Nelle note al canto successivo, Peter Weiss introduce un frammento altrettanto significativo che mette in evidenza come le difficoltà che emergono dallo studio del personaggio siano conseguenza diretta del valore allegorico di Virgilio nella Commedia:

Dort wo das bewusst und absichtl. Böse herrscht vermag auch die Vernunft nichts auszurichten.

Vergil ist der Räpresentant der menschl. Vernunft ${ }^{19}$

Die Machtlosigkeit der intellektuellen Kräfte angesichts des willentlich Bösen ${ }^{20}$ (Müllender 2007: 345).

I termini con cui viene problematizzata la possibilità di entrare a Dite coincidono con le espressioni abitualmente impiegate da Weiss per difendere la necessità di comprendere Auschwitz, realtà normalmente confinata allo spazio dell'inimmaginabile e dell'irrappresentabile ${ }^{21}$ :

I8. "A Virgilio: non lasciarmi solo mi sento così disorientato 8/ıoo | qui c'è una scena ancora non del tutto chiara: Virgilio entra nella città di Dite, dibatte lì, ma Dante non viene a sapere di cosa si parli Forse può dubitare di Virgilio lo tradisce Virgilio davanti ai cittadini?" - Le traduzioni dei frammenti dello schema di lavoro sono mie. Il testo originale può consultarsi in Müllender (2007: 333-373). L'originale si conserva in PWA 225I.

19. La frase centrale del frammento è una cita letterale tratta da uno degli articoli che lo scrittore svedese Olof Lagercrantz pubblica nel giornale Dagens Nyheter tra i mesi di marzo e maggio del 1963 . È proprio la lettura di questi articoli a suggerire a Weiss l'idea di scrivere un dramma sulla Divina Commedia.

20. "Dove domina il male cosciente e intenzionale nemmeno la ragione riesce a concludere qualcosa. | Virgilio è il rappresentante della ragione umana |'L'impotenza delle forze intellettuali nei confronti del male intenzionale".

2I. Le riflessioni attorno alla rappresentabilità di Auschwitz sono indissolubilmente legate al DC-Projekt fin dalla sua ideazione. Già le prime allusioni al progetto che troviamo nei Quaderni di appunti (Notizbücher) dell'autore confermano questa circostanza: "Zuerst dachte ich, es ließe sich nicht beschreiben, doch da es Taten wird, von Menschen begangen, Menschen auf diese Erde. Lange glaubte ich, es sei unmöglich - 2 Jahrzehnte vergangen. Eine neue Generation, die nichts davon weiß" [All'inizio pensai che non si potesse descrivere, ma sono stati crimini commessi da uomini, da uomini su questa terra. Per lungo tempo pensai che era impossibile - sono passati due decenni, una nuova generazione, che non sa niente di ciò] (NB I, p. 226, traduzione mia). Il progetto Divina Commedia funge quindi come catalizzatore e Peter Weiss trova precisamente in Dante, il modello che gli permette di assumere la responsabilità di comprendere e rappresentare Auschwitz: "Es hieß ja lange, es sei diesen Dingen mit Worten beizukommen. Diese Dinge waren ebenso unfaßbar, wie es für Dante damals die Abgründe der Hölle waren. Un dann kommen wir doch wieder dazu, daß gerade dieses scheinbar Unfaßbare beschrieben werden muß, so genau wie möglich. [...]. Dante begab sich in diese Vision des Schreckens hinein. [...]" 
Jetzt der Einwand: aber dies ist von Menschen erbaut ersonnen und ausgeführt

deshalb auch möglich heranzukommen es zu sehen und zu durchschauen deshalb vielleicht nicht ein Engel sondern Eintritt durch eigene Kraft Oder: der Engel blickt nicht an, meint damit, er tut dies nicht für ihn persönlich,

son dern [sic] für den Menschen (sottolineato nell'originale) nur dem Menschen ist est möglich, das vom Menschen ersonnene zu verstehen ${ }^{22}$ (Müllender 2007: 345).

Osserviamo come l'impotenza di Virgilio non significhi per l'autore la sconfitta definitiva della ragione ma diventi piuttosto espressione di un fallimento episodico che solo si può superare attraverso la ragione stessa. La negazione della soluzione dantesca - l'arrivo, nel canto IX dell'Inferno del messo celeste, essere sovraumano che vince la resistenza dei diavoli e apre al pellegrino le porte di Dite con una semplice "verghetta" (If. IX 90) - viene dunque compensata tramite la dichiarazione della fiducia nelle possibilità umane di fare i conti con l'orrore di Auschwitz. Nello schema relativo ai canti successivi, Virgilio recupera infatti il ruolo che aveva in precedenza e Peter Weiss annota i momenti della Commedia in cui agisce da protettore del pellegrino. Quando Dante vuole abbracciare i suoi connazionali nel canto XVI dell'Inferno, per citare solo uno degli esempi che confermano questa circostanza, Virgilio lo avverte del rischio: Guido Guerra, Aldobrandi e Rusticucci sono i suoi assassini in potenza. Ciononostante, l'ultima allusione al personaggio che troviamo nello schema di lavoro mette di nuovo in discussione il suo ruolo: "die frage ist ob Vergil überhaupt notwendig ist" [la domanda è se Virgilio sia davvero necessario] (Müllender 2007: 36I), scrive Weiss nelle note che studiano il canto XXXII.

L'ambigua caratterizzazione di Virgilio, di cui lo schema di lavoro è la prima manifestazione, è una costante della prima fase del DC-Projekt, dove il personaggio riceve un trattamento divergente in ognuna delle bozze che precedono la stesura della versione definitiva. A variare è soprattutto il grado di dipendenza che definisce il rapporto tra Virgilio e Dante così come il grado di complicità della presunta guida rispetto ai crimini della società infernale.

(Weiss 1968: 146) [Per molto tempo si è detto che era impossibile venire a capo di queste cose con le parole. Erano cose inconcepibili, come lo erano un tempo per Dante gli abissi dell'inferno. Ma eccoci di nuovo al punto: proprio ciò che apparentemente è inconcepibile deve essere descritto] (Weiss 2007: 49).

22. "Ora il fallimento: ma questo è stato costruito, ideato e riempito da uomini | per questo motivo è possibile avvicinarsi, vederlo e osservarlo | per questo motivo forse non un angelo ma entrata attraverso le proprie forze | Oppure: l'angelo non guarda Dante e pensa di fare qualcosa non solo per lui $\mid$ ma per gli uomini solo gli uomini possono comprendere ciò che è stato ideato da uomini" 
Risulta specialmente significativo che nel primo tentativo di riscrittura dell'Inferno, Virgilio sia concepito, tale e come ha dimostrato Müllender (2007: II2-I3), come la coscienza razionale - "erklärende Zweitestimme" (2007: II2) - di Dante. Entrambi i personaggi, uno alter ego dell'altro, sono i protagonisti di un monologo che si svolge nel primo canto della riscrittura e che riprende dalla Commedia la situazione di disorientamento iniziale:

D: ichwachte [sic] auf ineinem [sic] Zimmer / ich lag auf einem bett [sic] / durchdas [sic] offene fenster [sic] /hörte ich die geräusche [sic] einer stadt [sic] / da wusste ich nicht / in welcher Stadt ich mich befand / ich wusste nicht / warum ich hier war / und auch meinen Namen hatte ich vergessen / V: Mein Name ist Dante Alighieri / ich bin zurückgekehrt in eine Stadt / die ich vor drei Jahrzehnten verlassen hatte / Dort war das Todesurteil über mich verhängt worden / Dreissig Jahre lebte ich im Exil / D: Du sprichts von mir / du gibtsmir [sic] einen Namen zurück / und eine Vergangenheit ${ }^{23}$ (Müllender 2007: 367).

Il Virgilio protagonista dei tre canti che si conservano di questa versione, che Müllender definisce precisamente come un dramma lirico (cfr. Müllender 2007: 104-136), non contiene nessun tratto di ambiguità. Il personaggio rappresenta invece un appoggio fondamentale per Dante che solo grazie a Virgilio riuscirà a superare la sua amnesia iniziale e a sviluppare, indipendentemente da lui, un'attitudine e un pensiero critici. Nella versione in prosa, che conosciamo come "bozza d'Aischmann" (Cfr. Müllender 2007: 137-I50) i protagonisti sono Daniel Aischmann e Gil, nomi germanizzati che sostituiscono rispettivamente Dante e Virgilio. Il ruolo privilegiato assunto dalla descrizione della società infernale, che diventa per la prima volta in maniera esplicita la Germania del dopoguerra, a scapito della dimensione soggettiva di ricerca identitaria che era il nucleo del dramma lirico, influisce nella caratterizzazione dei personaggi principali. Daniel è diventato in questa versione un personaggio autonomo che si rivela capace fin dal primo momento di razionalizzare le esperienze del passato e di opporsi ai rimproveri degli abitanti della città di Dite. Anche la caratterizzazione del personaggio che funge da guida subisce delle modifiche con rispetto al dramma lirico. Gil è presentato in questo caso come un amico d'infanzia di Daniel che è diventato complice dei criminali durante l'assenza del protagonista.

Virgilio scompare infine in quella che Müllender chiama la "bozza di Giotto” (Müllender 2007: 154-I96) precisamente perché la funzione di guida

23. "D: Mi svegliai in una stanza / disteso su un letto / attraverso la finestra / ascoltavo i suoni di una città / non sapevo in che città mi trovavo / non sapevo perchè ero qui / e anche il mio nome avevo dimenticato. / V: Il mio nome è Dante Alighieri / sono tornato in una città / che avevo lasciato trent'anni fa / Lì si era approvata la mia sentenza di morte / Ho vissuto trent'anni nell'esilio./ D: Parli al mio posto / mi concedi un nome / e un passato.". 
viene ora assunta da Giotto, evidenziando la problematicità della resignificazione cui è sottoposto il personaggio dantesco.

L'Inferno unisce come in un montaggio scene e motivi provenienti dalle diverse bozze, per cui la dimensione soggettiva del dramma lirico convive nella versione definitiva con elementi di critica politico-sociale. Anche la caratterizzazione dei personaggi è il risultato di una fusione apparentemente disordinata di attributi che vengono recuperati dai testi precedenti. Di conseguenza, Virgilio diventa nell'Inferno un personaggio così ambiguo da rifuggire qualsiasi interpretazione definitiva. Considero che la sua mutevolezza sia sintomo delle difficoltà di definire il ruolo che deve assumere la ragione nello scenario del dopo Auschwitz, per cui la coincidenza che si intuisce tra determinate attitudini del personaggio di Virgilio e il concetto di ragione strumentale non possa essere accidentale ma risultato piuttosto di un dialogo probabilmente non del tutto consapevole con le riflessioni di Adorno e Horkheimer.

Descriviamo brevemente le caratteristiche che definiscono Virgilio nell'Inferno weissiano, soffermandoci sul rapporto che instaura con il protagonista e identificando i punti di contatto con la Dialektik der Aufklärung. Virgilio è l'unico personaggio del dramma che, a eccezione di Dante, compare sia nella cornice sia all'interno della rappresentazione metateatrale, esercitando una funzione strutturale fondamentale. È proprio lui a fornire i dati biografici del personaggio Dante che, all'inizio dell'opera, si ritrova muto su un podio:

Er den wir Dante nennen ist in dieser Stadt geboren / verurteilt zu Verbrennung floh er vor langen Zeiten / und seinem Aussehn nach ist es ihm nicht / wie uns hier wohl ergangen ${ }^{24}$ (Weiss 2008: 26-27).

Le informazioni fornite da Virgilio, unico dei presenti a riconoscere Dante, fungono poi da canovaccio dello spettacolo, per cui sono proprio i suoi interventi a consentirne lo svolgimento. Una volta iniziato "Das Spiel" [la messinscena] (Weiss 2008: 39), il personaggio di Virgilio appare paradossalmente sia in qualità di guida di Dante che come corresponsabile dei misfatti della società infernale, senza che sia possibile identificare nel testo un momento di rottura atto a spiegare questa contraddizione in termini di tradimento. È il suo significato allegorico originale che ne dà una spiegazione e l'indefinibilità di Virgilio diventa così specchio delle incertezze dell'autore, che si serve del personaggio per inserirsi nel dibattito più intenso del dopoguerra, cosciente del fatto che, dopo la frattura di Auschwitz, si impone un ripensamento delle fondamenta della tradizione filosofica e culturale occidentale che hanno consentito la barbarie, e, concretamente, del concetto stesso di ragione. "Au-

24. "Costui che noi chiamiamo Dante è nato in questa città / Condannato al rogo fuggi tanti anni or sono /e a giudicare dal suo aspetto non se l'è passata bene / come noi qui". 
schwitz ha dimostrato inconfutabilmente - scriverà Adorno nella Dialettica Negativa (1975: 330) - il fallimento della cultura. [...] Tutta la cultura dopo Auschwitz, compresa la critica urgente a essa, è spazzatura".

Da questo punto di vista possiamo mettere in relazione l'atteggiamento subdolo del personaggio, che subisce rispetto al modello un evidente processo di trasformazione assiologica, e, in concreto, di devalorizzazione (Genette 1989: 432), con il processo di perversione della ragione che, secondo le riflessioni contenute nella Dialettica dell'Illuminismo (1947) di Adorno e Horkheimer, avrebbe portato al fallimento del progetto stesso dell'Illuminismo, di cui precisamente Auschwitz sarebbe la conseguenza più evidente e drammatica.

Scritto con l'obiettivo di comprendere perché l'umanità, invece di entrare in uno stadio realmente umano, abbia invece generato l'istituzionalizzazione della barbarie (Adorno e Horkheimer 2009: 5I), il primo saggio del volume identifica le cause di questa involuzione nella potenzialità autodistruttiva della ragione, ma anche nelle contraddizioni interne al programma illuministico di emancipazione, che nasce, dal loro punto di vista, sotto il segno del dominio. Il conseguente processo di razionalizzazione della natura ha come risultato fondamentale la strumentalizzazione del sapere, per cui lo sviluppo della scienza moderna riduce il pensiero a ripetizione tautologica, a semplice giustificazione dell'ordine esistente (Adorno e Horkheimer 2009: 80).

Anche gli interventi di Virgilio si limitano a celebrare senza sottoporre a riflessione, le peculiarità che definiscono la società infernale. Nonostante lo stesso Virgilio neghi il proprio ruolo di guida nel canto III - "Dort ging er auserwählt und anvertraut / nobelster Führung / doch als er aufwachte war ihm / der Wegweiser verschwunden" [Là se ne andava eletto e affidato / alla più nobile delle guide / ma quando si svegliò era scomparso chi gli indicava la via] (Weiss 2008: 35) -, Dante si mostra dipendente da lui e, come nella Commedia, si rivolge al poeta per cercare spiegazioni e protezione. Virgilio assume inoltre il compito di mediare tra Dante e i funzionari infernali almeno fino al canto XIX, momento in cui il personaggio si mostra capace per la prima volta di dialogare direttamente con i rappresentanti di Dite.

I chiarimenti di Virgilio si trasformano però in discorsi apologetici della città infernale, realtà che viene esaltata precisamente in quanto "Reich der Mitte" [Regno di mezzo] da cui "strahlt Kraft nach allen Seiten aus" [si irradia forza in ogni direzione] (Weiss 2008: II9), paradiso del progresso, della libertà e della giustizia. I celebri versi nei quali il poeta latino nell'ipotesto riesce a piegare la resistenza di Caronte (If. III, 95-96) e di Minosse (If. V, 23-24), alludendo alla legittimazione divina del viaggio di Dante, vengono modificati da Peter Weiss che li fa diventare espressione delle illimitate possibilità che la città di Dite offre all'individuo: 
Sieh doch wie offen es ist hier / nicht bindet dich / keine Vergangenheit ist die in Weg / und alles was du willst / das steht dir frei zu tun / Sieh dich doch um / sieh wies hier lebt / wie alle gehn wohin sie wollen / und jeder spricht wies ihm gefällt ${ }^{25}$ (Weiss 2008: 46-49, corsivo mio $^{26}$ ).

Tutti gli sforzi di Virgilio sono orientati a persuadere il poeta ad accettare la legittimità delle norme che reggono la società. Anche in questo senso Virgilio agisce in maniera analoga alla ragione strumentale in quanto rassegnazione del pensiero alla produzione della conformità (Adorno e Horkheimer 2009: 88), per cui il pensiero critico si trasforma in affermazione dell'esistente.

Disposto a conseguire a tutti i costi l'integrazione di Dante, Virgilio reagisce con violenza quando il pellegrino si mostra reticente alle sollecitazioni dei funzionari infernali: nel canto IX costringe Dante ad accettare la decorazione che gli offre Pluto e nel canto XIX è lui stesso, come appare dalla relativa didascalia, a mettere la penna in mano al personaggio per firmare nel "goldnen Buch der Stadt" [libro d'oro della città] (Weiss 2008: I3I).

In questo modo Virgilio contraddice apertamente sia il ruolo che svolge nell'ipotesto sia quello che Weiss pareva avergli conferito nello schema di lavoro. In effetti, i momenti in cui il personaggio protegge Dante dai pericoli dell'inferno diventano nella versione definitiva occasione per rendere manifesta la complicità di Virgilio con la società dei carnefici: nel canto XVI Virgilio, invece di celare gli occhi al suo compagno per proteggerlo dallo sguardo pietrificante di Medusa, lo spinge con un calcio verso il mostro. Paradossalmente sarà poi lo stesso Virgilio, dopo aver dichiarato la propria impotenza - "Ich kann jetzt nichts mehr für dich tun / Du bist hergekommen aus freien Willen um dich ihnen zu stellen" [Ora non posso fare più nulla per te / Sei venuto di tua spontanea volontà / per consegnarti a loro] (Weiss 2008: 219) - a nascondersi la faccia tra le braccia per non assistere all'aggressione finale del canto XXXI, dove Dante è imprigionato con una rete, prima che la detonazione di ciò che sembra essere una bomba atomica concluda il dramma.

Alla fine del canto XXIV il personaggio di Latini "In einer Drehung nimmt er sich die Maske ab und zeigt sich als Vergil" [facendo una giravolta si toglie la maschera / e si palesa essere Virgilio] (Weiss 2008: I69). Ripensando al contenuto del canto, lo svelamento di questa circostanza - un altro degli aspetti del dramma di Weiss che l'editore Braun reputava incomprensibili- si rivela enormemente significativo. In bocca del Maestro Latini, altro personaggio profondamente ambiguo, la profezia dell'esilio (If. XV, 6I-78)

25. "Guarda come è aperto qui / nulla ti lega /nessun passato ti sta tra i piedi / e tutto ciò che vuoi / puoi farlo liberamente / Guardati un po' attorno / guarda come si vive qui /come tutti vanno dove vogliono / e ciascuno parla come più gli piace".

26. Ė lo schema di lavoro a confermare che i versi del dramma derivano dai danteschi "Vuolsi così colà dove si puote ciò che si vuole / e più non dimandare", che già nello schema si trasformano in "er kann, was er will” [egli può, ciò che vuole] (Müllender 2007: 340). 
dell'originale diventa nella riscrittura occasione di legittimazione politica dell'esclusione razziale. La superiorità d'animo che Brunetto Latini riconosce immediatamente in Dante, "dolce fico" tra "lazzi sorbi" (If. XV, 65-66), viene reinterpretata dal personaggio del dramma in chiave di giustificazione della sua selezione, che paradossalmente non comporta la morte bensì la salvezza:

Ich liebte dich ich liebte dich weil du anders warst / als die Meisten / Ach ich liebte dich da ich schön früh sah / dass du ihnen überlegen warst im Geiste [...] / Weil ich nicht wollte dass du dich drängtest / mit den andern / du und die wenigen andern / die gleichwertig waren mit $\operatorname{dir}^{27}$ (Weiss 2008: 162-I65).

Del canto, che fornisce spunti di riflessione interessanti per studiare in che modo Weiss tematizza nel testo il proprio complesso di colpa servendosi del personaggio di Dante, ci interessa specialmente il fatto che è proprio Virgilio a realizzare questa selezione, ammettendo così una diretta responsabilità rispetto alle politiche di sterminio. Anche in questo senso troviamo punti di coincidenza con la tesi che regge il saggio della Dialettica dell'Illuminismo e che identifica nella vendetta della natura reificata dall'Illuminismo l'origine stessa della barbarie. Paradossalmente, la perversione della ragione e la sua strumentalizzazione possono essere superate, e uscire dallo stato di regressione, solamente attraverso un esercizio autoriflessivo portato avanti dalla ragione stessa (Adorno e Horheimer 2009: 3I). La fiducia nella possibilità dell'Illuminismo di trovare una via d'uscita diversa da quella del dominio della tecnica e della razionalità strumentale corrobora l'idea che "nonostante il suo carattere radicale, questa critica [quella di Adorno e Horkheimer] apparteneva quindi anch'essa, nonostante tutto, alla tradizione dell'Illuminismo" (Traverso 2004: II9). Anche Peter Weiss, quasi vent'anni dopo la prima pubblicazione della Dialettica dell'Illuminismo e in una società ormai pre-postmoderna che prefigura l'accettazione aproblematica della distruzione della ragione, cerca disperatamente, come Adorno e Horkheimer, "di salvare il nocciolo emancipatore della razionalità occidentale dalle derive dell'Aufklärung" (Traverso 2004: II8). L'ambiguità di Virgilio diventa, come annunciavamo, espressione di questa contraddizione, per cui il suo personaggio oltre a rappresentare la perversione dell'Illuminismo occidentale rivendica la sopravvivenza del ruolo chiarificatore della ragione, unico strumento con cui riuscire a interpretare non solo Auschwitz ma anche la realtà infernale posteriore che viene, non a caso, delineata da Weiss in termini grotteschi. Indicativo è, da questo punto di vista, l'intervento con cui Virgilio, nel canto XI, ribadisce la comprensi-

27. “Ti amavo ti amavo perché eri diverso / dai più / Ah ti amavo poiché fin dall'inizio vidi / che eri superiore a loro in spirito / [...] / Perché non volevo che finissi nella calca / con gli altri / tu e i pochi altri / che ti erano pari in valore". 
bilità della città di Dite, luogo che allude sia al lager sia alla società tedesca degli anni sessanta:

Weisst du denn nicht / Es ist doch unsre Stadt / Kennst du den Fluss nicht wieder / Heisst dich Styx / Und unsre Stadt heisst Dis / Sie ist von Lebenden erbaut / und wenn du diese Stadt siehst / lebst du auch / Wie könnte das denn unverständlich sein / Wie könnte irgendetwas unverständlich sein / das Lebende erdachten und erbauten ${ }^{28}$ (Weiss 2008: 84-85).

Un altro aspetto del dramma - che mi limito ad accennare a modo di conclusione e come spunto per successive analisi - rafforza l'ipotesi che rivendica l'importanza della Dialektik der Aufklärung come testo sul cui sfondo si articola la costruzione di Virgilio. Mi riferisco al trattamento drammatico che riceve il controverso Ulisse dantesco nell'Inferno weissiano, anch'egli sottoposto, come Virgilio, a un processo di devalorizzazione (Genette 1989: 432). Pur rimanendo fedele al ritratto che si estrae dalla Commedia, dove il consigliere fraudolento viene punito per l'astuzia con cui convince i compagni ad intraprendere "il folle volo" (If. XXVI I25), Weiss rifiuta il tradizionale elogio umanistico del personaggio e della famosa "orazion picciola" (If. XXVI I22).

Se, già nello schema di lavoro, Weiss percepiva la duplice dimensione dell'Ulisse dantesco e stentava a identificarlo con un referente extra-testuale a lui contemporaneo ${ }^{29}$, nella versione definitiva incontriamo un General Odysseus privo di ogni possibile connotazione positiva:

Ihr Heldensöhne zeigt dass ihr bereit seid /durch hunderttausend Gefahren zu gehn / wie eure Väter / Kämpft um die Werte / die unsre Schöpfung sind / Wie Kinder sollt ihr sein / die sich der weisen Führung / Ihrer Väter anvertraun / Und wisst dass ihr unsere Befehle / nach besten Kräften / vollständig / gewissenhaft und unverzüglich / auszuführen habt ${ }^{30}$ (Weiss 2008: 202-205).

L'"orazion picciola” originale è evocata e capovolta, l'ambivalenza eliminata. È forse il primo excursus della Dialektik der Aufklärung (Odysseus oder Mythos und Aufklärung) a influire sulla caratterizzazione definitiva del personaggio weissiano. Il rapporto di dominio tra il generale e gli ascoltatori coincide, in

28. "Ma come non lo sai / suvvia è la nostra città / non riconosci il fiume /Ma sì Stige è il suo nome /E la nostra città si chiama Dite / È stata costruita da esseri vivi /e se vedi questa città allora sei vivo anche tu / Come può essere incomprensibile / Come può essere incomprensibile una qualsiasi cosa / pensata e costruita da esseri vivi".

29. "Noch problematisch - scrive Weiss - ob Odysseus der hitler-typ oder alter ego für Dante" [Ancora problematico se Ulisse è il tipo-Hitler o alter ego di Dante] (Müllender 2007: 357).

30. "O figli d'eroi / mostrate di essere pronti ad attraversare centomila perigli / come i vostri padri / Lottate per i valori che abbiamo creato noi / Dovete essere come bambini / che si affidano alla saggia guida dei padri / umili e devoti / E sappiate / che siete tenuti a eseguire / fino ai limiti del possibile / interamente / coscienziosamente e immediatamente i nostri ordini". 
effetti, con la descrizione che i filosofi fanno dell'eroe, in particolare nell'episodio delle sirene, espressione delle dinamiche di schiavitù della civiltà occidentale. Senza dettagliare l'argomentazione che porta Adorno e Horkheimer a rapportare l'astuzia di Ulisse con il paradigma della ragione strumentale, conviene ricordare che precisamente il termine astuzia (Die List) viene impiegato da Weiss in un passaggio-chiave dell'Istruttoria (Weiss: 1991: 39) che, come ha notato M. Meyer, funge da "Gedächtnisraum" (2000: III), spazio di memoria che rievoca indirettamente sia il contenuto della Dialektik der Aufklärung che Primo Levi, e tramite lui, Dante e il canto di Ulisse:

Überleben konnte nur der Listige [...] / Die Unfähigen / Die Trägen im Geiste / die Milden / die Verstörten und Unpraktischen / die Traurenden und die / die sich selbst bedauerten / wurden zertreten ${ }^{31}$

Queste brevi considerazioni sulle modificazioni a cui è sottoposto il personaggio di Ulisse appoggiano l'ipotesi che vincola Virgilio al concetto di ragione strumentale sviluppato nella Dialettica dell'Illuminismo, rivelando come anche altri momenti del dialogo che Weiss stabilisce con Dante sembrano attingere ai contenuti dell'opera. Entrambi i personaggi si costruiscono inoltre mediante un processo di attualizzazione negativa dei contenuti originali, svelando come una lettura attenta delle modalità di appropriazione dei materiali dell'ipotesto scopra delle dimensioni inattese dell'Inferno di Peter Weiss che non possono far altro che arricchire la nostra interpretazione.

\section{BIBLIOGRAFIA}

Adorno, Th.W., 1975, Dialettica negativa, Einaudi, Torino.

Adorno, Th.W., Horkheimer, M., 2009, Dialéctica de la Ilustración, introducción y traducción de Juan José Sánchez, Madrid, Trotta.

Alighieri, D., 2006, La Divina Commedia, annotata e commentata da Tommaso di Salvo, con illustrazioni, Milano, Zanichelli.

Arqués, R., 2009, "Dante nell'inferno moderno: la letteratura del dopo Auschwitz" Rassegna europea di letteratura italiana 33, pp. 87-Io9.

Brunner, B., 2002, "Peter Weiss und das Inferno. Über ein unveröffentliches Stück, die Ermittlung und das Verhältnis zu Nachkriegsdeutschland - eine Auseinandersetztung mit den Interpretationen von Christoph Weiß”, Peter Weiss Jahrbuch, II, pp. 56-83.

Casadei, A., 2013, Dante oltre la Commedia, Bologna, Il Mulino.

Castellari, M., 2008, "Dei molti inferni. La Shoa, Dante e Peter Weiss" in Weiss, P., Inferno. Testo drammatico e materiali critici, edizione bilingue a cura di Marco Castellari, Milano, Mimesi Edizioni, pp. 237-26I.

3I. "Sopravvivere poteva solo l'astuto / [...] /gli incapaci / i lenti di spirito / i clementi / i turbati e poco pratici / coloro che portano il lutto e coloro / che hanno compassione di sé stessi / erano calpestati”. 
Dallapiazza, M., 2oıo, "L'inferno è il presente, non l'aldilà. Dante nella letteratura tedesca dell'esilio", in La ricezione di Dante Alighieri: impulsi e tensioni. Atti del convegno internazionale all'Università di Urbino, 26 e 27 Maggio 20I0, a cura di Unfer Lukoschik, R., e Dalla Piazza, M., pp. I29-I43.

Genette, G., 1989, Palimpsestos. La literatura en segundo grado, traducción de Celia Fernández Prieto, Madrid, Taurus.

Heinrich, C., 2006, "Zur Kenntlicheit ent-stellt. Vergleichende Analyse des Librettos von Johannes Kalitzke zur Operinszeniertung Inferno und des Texts des gleichnahmigen Theaterstücks von Peter Weiss", Peter Weiss Jahrbuch, I5, pp. 69-96.

Kretschmann, T., 20I2, "Nachträgliche Standortbestimmung. Zu Peter Weiss' postum veröffentlichtem Drama Inferno (1964/2003) und seiner Uraufführung am Badischen Staatstheater Karlsruhe (2008), Höllenmaschine / Wunschapparat”. Analysen ausgewählter Neubearbeitungen von Dantes Divina Commedia. Bielefeld: transcript, pp. 25-77.

Kuon, P., I993, "Lo mio maestro e'l mio autore". Die produktive Rezeption der Divina Commedia in der Erzähllitertur der Moderne, Anakleta Romanica Heft 53, Vittorio Klostermann, Frankfurt am Main.

Kuon, P., 1997, “...dieser Portalheilige zur abendländischen Kunst... Zur Rezeption der Divina Commedia bei Peter Weiss, Pier Paolo Pasolini und anderen”, Peter Weiss Jahrbuch, 6, pp. 42-67.

Lagercrantz, O., I983, Scrivere come Dio. Dall'Inferno al Paradiso, traduzione italiana di Carmen Giorgietti, Cima, Marietti, Casale Monferrato.

Meyer, M., 2009, "Bergpredigt - Dante - Odysseus - Levi. Ein Intertextueller Kommentar zu zwei Sätzen im Gesang vom Lager in Peter Weiss' Ermittlung”, Peter Weiss Jahrbuch, 9, pp. IO2-II4.

Müllender, Y., 2007, Peter Weiss' Divina Commedia-Projekt (1964-I969) ...läßt sich dies noch beschreiben... Prozesse der Selbstverständigung und der Gesellschaftskritik, Röhring Universitätsverlag, St. Ingbert.

Rector, M., 1997, "Sechs Thesen zur Dante-Rezeption bei Peter Weiss", Peter Weiss Jahrbuch, 6, pp. IIO-II5.

Taterka, Th., 1999: Dante Deutsch: Studien zur Lagerliteratur, Berlin, Erich Schmidt.

Traverso, E., 2004, Auschwitz e gli intelettuali. La Shoa nella cultura del dopoguerra, Bologna, Il Mulino.

Weiß, C., 2000, Auschwitz in der geteilten Welt. Peter Weiss und die Ermittlung im Kalten Krieg, Röhrig Univeristätsverlag.

—, 2008, "Postfazione all'edizione tedesca", in Weiss 2008: 263-285.

Weiss, P., 1968, Rapporte, Frankfurt am Main, Suhrkamp Verlag.

—, 1982, Notizbücher 1960-I97I, Frankfurt am Main, Suhrkamp Verlag.

—, I99I, Die Ermittlung, Oratorium in II Gesängen, Frankfurt am Main, Suhrkamp Verlag.

—, 2007, Inferni, Auschwitz, Dante, Laoconte, a cura di Clemens-Carl Härle, traduzione di Anna Persa, Napoli, Cronopio.

—, 2008, Inferno. Testo drammatico e materiali critici, ed. bilingue a cura di Marco Castellari, Milano, Mimesi Edizioni.

Weymann, U., 2008, "Lasst alle Zweifel fahren. Die Uraufführung des Inferno in der Inszenierung von Thomas Krupa", Peter Weiss Jahrbuch, I7, I67-I73.

Wöhl, J., I997, Intertextualität und Gedächnisstiftung: Die Divina Commedia Dante Alighieris bei Peter Weiss und Pier Paolo Pasolini, Frankfurt am Main, Lang. 